\title{
Adaptively Multi-Objective Adversarial Training for Dialogue Generation
}

\author{
Xuemiao Zhang ${ }^{1 *}$, Zhouxing Tan ${ }^{1 *}$, Xiaoning Zhang ${ }^{2}$, Yang Cao ${ }^{4}$ and Rui Yan ${ }^{3 \dagger}$ \\ ${ }^{1}$ School of Software \& Microelectronics, Peking University \\ ${ }^{2}$ Changchun Institute of Optics, Fine Mechanics and Physics, Chinese Academy of Sciences \\ ${ }^{3}$ Wangxuan Institute of Computer Technology, Peking University \\ ${ }^{4}$ SenseTime Research \\ \{zhangxuemiao, tzhx, ruiyan\}@pku.edu.cn, xiaoningzhang42@gmail.com, caoyang@ sensetime.com
}

\begin{abstract}
Naive neural dialogue generation models tend to produce repetitive and dull utterances. The promising adversarial models train the generator against a well-designed discriminator to push it to improve towards the expected direction. However, assessing dialogues requires consideration of many aspects of linguistics, which are difficult to be fully covered by a single discriminator. To address it, we reframe the dialogue generation task as a multi-objective optimization problem and propose a novel adversarial dialogue generation framework with multiple discriminators that excel in different objectives for multiple linguistic aspects, called AMPGAN, whose feasibility is proved by theoretical derivations. Moreover, we design an adaptively adjusted sampling distribution to balance the discriminators and promote the overall improvement of the generator by continuing to focus on these objectives that the generator is not performing well relatively. Experimental results on two real-world datasets show a significant improvement over the baselines.
\end{abstract}

\section{Introduction}

The end-to-end neural systems [Serban et al., 2016; Luan $e t$ al., 2016] based on SEQ2SEQ framework [Sutskever et al., 2014] and using the maximum likelihood estimation (MLE) objective have been extensively studied because of its ability to generate unseen conversations and high scalability. But the dialogue responses generated by these models tend to be generic, dull, repetitive [Tao et al., 2018; Li et al., 2016; Zhang et al., 2018] in practice. Recently, sequence generative adversarial nets (SeqGAN) [Yu et al., 2017] apply the promising adversarial training method of GANs [Goodfellow et al., 2014] on sequence generation task by employing policy gradient and Monte Carlo (MC) search. AdverREGS [Li et al., 2017] employs adversarial training to actual dialogue generation task and trains the generator against a real-fake discriminator. DP-GAN [Xu et al., 2018] and DAL [Cui et

\footnotetext{
${ }^{*}$ Equal contribution.

${ }^{\dagger}$ Corresponding author: Rui Yan (ruiyan@pku.edu.cn)
}

al., 2019] try to increase the diversity of the generated responses by designing a fine-grained diversity discriminator and utilizing the duality between query and response generation, respectively.

But in fact, assessing dialogue responses should consider many aspects of linguistics such as diversity, fluency, syntax habits of human, and so on. However, although a welldesigned discriminator can make the generator perform well in the corresponding expected aspect, other aspects are often ignored. We notice that the multi-adversarial framework, denoted MDGAN, that extends GANs to multiple discriminators has achieved promising results on image generation tasks. Many models in MDGAN [Durugkar et al., 2017; Neyshabur et al., 2017; Albuquerque et al., 2019] use their specially designed methods to weight the sum of the losses of image discriminators to optimize the image generator overall.

We hope the generator can cover multiple linguistic aspects to achieve overall improvement and perform relatively well in all aspects. Intuitively, we can view dialogue generation as a multi-objective optimization problem [Deb, 2001; Albuquerque et al., 2019]. Each objective is designed for a linguistic aspect, and a corresponding discriminator provide guidances towards this objective, thereby optimizing the generator towards multiple objectives. However, this idea faces challenges. The main challenge is to innovatively propose a theoretically proven and practically effective framework that extends the original adversarial dialogue generator optimization problem to a multi-objective optimization problem, where each objective is defined by policy gradient. The second is to explore how to design and combine various linguistic objectives, and to deal with the problem of asynchronous optimization of different objectives during training.

In this paper, we propose the adaptively multi-objective adversarial dialogue generation framework using policy gradient, called AMPGAN, to push the generator $G_{\theta}$ to achieve overall improvement. AMPGAN models dialogue generation as a stochastic policy in reinforcement learning (RL) and directly performs gradient policy update to generate discrete dialogue utterances, and is formalized as the multiobjective optimization problem by framing the simultaneous maximization of rewards received from multiple discriminators. We design multiple objectives of linguistics for guiding $G_{\theta}$ of AMPGAN: indistinguishable from the human utterances, high syntactic score and diversity. We propose mul- 
tiple corresponding discriminators and an adaptively adjusted sampling distribution to dynamically organize them to participate in adversarial training. Through the adaptive distribution, AMPGAN can focus on the aspects that $G_{\theta}$ not perform well relatively. When $G_{\theta}$ is still weak and have much room to improve in aspect $a_{i}$, AMPGAN will adaptively increase the probability that the corresponding $D_{i}$ is selected to participate in adversarial training, to motivate $G_{\theta}$ to optimize towards $a_{i}$; Conversely, when $G_{\theta}$ can easily deceive the discriminator $D_{j}$ of aspect $a_{j}$, AMPGAN will adaptively reduce its probability, and pay more attention to other relatively weak aspects. Thereby, $G_{\theta}$ can cover multiple aspects and solve the asynchronous optimization problem.

Our contributions are summarized as: (1) We reframe the dialogue generation as a multi-objective optimization problem and propose the novel multiple adversarial generation framework (AMPGAN) to improve the generator towards multiple objectives of linguistics and provide the theoretical derivation and proof; (2) We explore how to choose and combine different discriminators, and design an adaptively adjusting sampling distribution to balance all discriminators; (3) We conduct ample experiments on two datasets, and experimental results show the effectiveness of AMPGAN framework.

\section{Methodology}

We propose the adaptively multi-adversarial dialogue generation framework using policy gradient, called AMPGAN, to push the dialogue generator to improve towards multiple objectives based on linguistic apects, as shown in Figure 1.

\subsection{The AMPGAN Framework}

Given a dialogue query utterance $x=\left\{x_{i}\right\}_{i=1}^{m}$ of $m$ words, the generator $G_{\theta}$ needs to produce a response $y=\left\{y_{j}\right\}_{j=1}^{n}$ of $n$ words, where $x_{i}, y_{j} \in \mathcal{T}$ (the word vocabulary). AMPGAN conducts gradient policy update directly by modeling $G_{\theta}$ defined by a SEQ2SEQ model as a stochastic policy in reinforcement learning (RL). Each discriminator in AMPGAN is designed to judge on the complete generated sequence according to a linguistic objective, and reports the RL reward which is passed back to the intermediate state-action steps using MC search. Note that all discriminators $\left\{D_{i}\right\}_{i=1}^{N}$ do not share parameters. We extend and divide the types of discriminators into three categories, binary classification task $D_{C}$, regression task $D_{R}$ and direct rule scorer $D_{I}$. Among them, $D_{C}$ and $D_{R}$ are parameterized models that need training, and $D_{I}$ is a set of scoring logical rules which can be understood as a fully trained and completely correct discriminator.

More formally, $G_{\theta}$ and $\left\{D_{i}\right\}_{i=1}^{N}$ play the following minimax optimization game:

$$
\begin{array}{r}
\min _{G_{\theta}} \max _{\left\{D_{i}\right\}_{i=1}^{N}} V\left(G_{\theta},\left\{D_{i}\right\}_{i=1}^{N}\right)=\mathbb{E}_{D \sim \pi(Q)}\left(\mathbb{E}_{\boldsymbol{x} \sim p_{d}(\boldsymbol{x})}\right. \\
\left.\log (D(\boldsymbol{x}))+\mathbb{E}_{\boldsymbol{z} \sim p_{z}(\boldsymbol{z})} \log \left(1-D\left(G_{\theta}(\boldsymbol{z})\right)\right)\right)
\end{array}
$$

where the random variable discriminator $D$ obeys the distribution $\pi(Q)$ on the increment $Q$ of RL rewards. $\boldsymbol{x}$ is the ground truth data and $z$ is the query data drawn from a prior $p_{z}(\boldsymbol{z}) . G_{\theta}$ maps $\boldsymbol{z}$ to the fake responses which obeys the distribution $p_{g}$. Next we will provide formal theoretical analysis.

\section{Theoretical Analysis}

Firstly, we consider the optimization of multiple discirminators for any given $G_{\theta}$ and $\pi(Q)$ under the proposed AMPGAN framework. Note that since the rule scorer $D_{I}$ is constant, here we only need to consider the variable $D_{C}$ and $D_{R}$.

Proposition 1. For $G_{\theta}$ and $\pi(Q)$ fixed, the optimal discriminator $D_{i}$ is: $D_{i}{ }^{*}=p_{d}(\boldsymbol{x}) /\left(p_{d}(\boldsymbol{x})+p_{g}(\boldsymbol{x})\right)$.

Proof. According to the induced measure theorem [Somesh Das Gupta, 2000], the two expectations are equal: $\mathbb{E}_{\boldsymbol{z} \sim P_{\boldsymbol{z}}}[f(G(\boldsymbol{z}))]=\mathbb{E}_{\boldsymbol{x} \sim P_{G}}[f(\boldsymbol{x})]$. The objective function can be rewritten as below:

$$
\begin{array}{r}
V\left(G,\left\{D_{i}\right\}_{i=1}^{N}\right)=\int_{\boldsymbol{x}} \sum_{i=1}^{N} p_{D_{i}}\left(p_{d}(\boldsymbol{x}) \log D_{i}(\boldsymbol{x})\right. \\
\left.+p_{g}(\boldsymbol{x}) \log \left(1-D_{i}(\boldsymbol{x})\right)\right) d \boldsymbol{x}
\end{array}
$$

Let $A:=\sum_{i=1}^{N} p_{D_{i}}\left(p_{d}(\boldsymbol{x}) \log D_{i}(\boldsymbol{x})+p_{g}(\boldsymbol{x}) \log \left(1-D_{i}(\boldsymbol{x})\right)\right)$ then $\operatorname{diag}\left(\left\{\frac{p_{D_{i}}\left(p_{d}(\boldsymbol{x})\left(D_{i}-1\right)^{2}+p_{g}(\boldsymbol{x}) D_{i}{ }^{2}\right)}{D_{i}{ }^{2}\left(D_{i}-1\right)^{2}}\right\}_{i=1}^{N}\right)$ is the Hessian matrix of $-A$, and all leading principle minors of the matrix are greater than 0 , thereby $-A$ is a convex function. So the maximum point $D_{i}^{*}$ of $\mathrm{A}$ is stationary points on $[0,1]$ :

$$
\begin{aligned}
\frac{\partial A}{\partial D_{i}} & =p_{D_{i}}\left(\frac{p_{d}(x)}{D_{i}}+\frac{p_{g}(x)}{D_{i}-1}\right)=0 \\
D_{i}{ }^{*} & =p_{d}(\boldsymbol{x}) /\left(p_{d}(\boldsymbol{x})+p_{g}(\boldsymbol{x})\right) \in[0,1]
\end{aligned}
$$

And $D_{i}^{*}$ is also the optimal point of $V\left(G,\left\{D_{i}\right\}_{i=1}^{N}\right)$ Q.E.D.

Secondly, we fix $D_{i}=D_{i}^{*}$ and find the optimal solution $G^{*}$ for the generator of the proposed AMPGAN framework.

Theorem 1. Let $C(G)=\max _{\left\{D_{i}\right\}_{i=1}^{N}} V\left(G,\left\{D_{i}\right\}_{i=1}^{N}\right)$, then $\min C(G)=-\log 4$.

Proof. Bring $D_{i}^{*}$ into the above formula:

$$
\begin{aligned}
& C(G)=\sum_{i=1}^{N} p_{D_{i}}\left[\mathbb{E}_{\boldsymbol{x} \sim p_{d}(\boldsymbol{x})} \log \left(\frac{p_{d}(\boldsymbol{x})}{p_{d}(\boldsymbol{x})+p_{g}(\boldsymbol{x})}\right)\right. \\
& \left.\quad+\mathbb{E}_{\boldsymbol{x} \sim p_{g}(\boldsymbol{x})} \log \left(\frac{p_{g}(\boldsymbol{x})}{p_{g}(\boldsymbol{x})+p_{d}(\boldsymbol{x})}\right)\right] \\
& \quad=\left(\sum_{i=1}^{N} p_{D_{i}}\right)\left(-\log 4+\left(K L\left(p_{d}(x) \| \frac{p_{d}(x)+p_{g}(x)}{2}\right)\right.\right. \\
& \left.\quad+K L\left(p_{g}(x) \| \frac{p_{d}(x)+p_{g}(x)}{2}\right)\right) \\
& \quad=-\log (4)+J S D\left(p_{d} \| p_{g}\right)
\end{aligned}
$$

when $p_{d}$ and $p_{g}$ are consistent, get the minimum value $-\log (4)$ Q.E.D.

Thirdly, we directly use a policy gradient of RL to optimize $G_{\theta}$, which can avoid the problem of differentiation difficulty [Yu et al., 2017] for discrete dialogue data in the traditional GAN naturally. Each $D_{i}$ in AMPGAN guides to improve $G_{\theta}$ by computing a specific reward signal on sequences generated by $G_{\theta}$ using MC rollout strategy. Specifically, starting from the current state $s=Y_{1: t-1}$, action $a=y_{t}$, using MC search until the complete sequence is generated, the search sequence set $M C^{G_{\theta}}\left(Y_{1: t} ; N\right)$ is obtained. And the value function $Q_{D_{i}}^{G_{\theta}}$ of single discirminator $D_{i}$ on this set is set to the average discrimination score as:

$$
Q_{D_{i}}^{G_{\theta}}\left(s=Y_{1: t-1}, a=y_{t}\right)=\frac{1}{M} \sum_{m=1}^{M} D_{i}\left(Y_{1: T}^{m}\right)
$$






Figure 1: The architecture of AMPGAN. Given queries, the generator generates fake responses. A discriminator will be sampled to compute the reward to optimize the generator according to the adaptively adjusted distribution. All discriminators are supervisedly trained by using true and fake responses.

where $Y_{1: T} \in M C^{G_{\theta}}\left(Y_{1: t} ; N\right), M$ is the simulations number of MC. When there is no intermediate reward, we can use the average value $J_{D_{i}}(\theta)$ assigned by the expectation of value function $Q_{D_{i}}^{G_{\theta}}$ to evaluate the policy $G_{\theta}$ [Sutton et al., 2000].

$$
J_{D_{i}}(\theta)=\sum_{y_{1} \in Y} G_{\theta}\left(y_{1} \mid s_{0}\right) Q_{D_{i}}^{G_{\theta}}\left(s_{0}, y_{1}\right)
$$

In AMPGAN framework, dialog generation is viewed as a multi-objective optimization problem. Formally, the generator solves the following multi-objective problem:

$$
\max \mathcal{J}(\theta)=\left[J_{D_{1}}(\theta), J_{D_{2}}(\theta) \ldots J_{D_{N}}(\theta)\right]^{T}
$$

Specifically, we design an adaptively adjusted distribution $D \sim \pi(Q)$ to organize the multiple objectives as:

$$
\max \mathcal{J}(\theta)=\mathbb{E}_{D \sim \pi(Q)} J_{D}(\theta)
$$

So that AMPGAN can continue to pay attention to the objectives that $G_{\theta}$ not performs well relatively and $G_{\theta}$ can be balanced and improved towards many objectives more stably.

Formally, when $G_{\theta}$ is weak in an objective, AMPGAN dynamically strengthens the adversarial training between $G_{\theta}$ and the corresponding $D_{i}$ by adaptively adjusting the sampling distribution $\pi(Q)=\left\{p^{(k)}\left(D=D_{i}\right)\right\}_{i=1}^{N}$ as:

$$
p\left(D_{i}\right)^{(k)}=\frac{1}{Z} p\left(D_{i}\right)^{(k-1)} \exp \left(\Delta Q_{D_{i}}^{G_{\theta}(k)}\right)
$$

where $Z=\sum_{i=1}^{N} p_{i}^{(k-1)} \exp \left(\Delta Q_{D_{i}}^{G_{\theta}(k)}\right)$ and $\Delta Q_{D_{i}}^{G_{\theta}(k)}=Q_{D_{i}}^{G_{\theta}(k)}-$ $Q_{D_{i}}^{G_{\theta}{ }^{(k-1)}}+\mathcal{N}(0, \sigma)$ and $\mathcal{N}(0, \sigma)$ is Gaussian noise, and initial $\pi(Q)$ is $p^{(0)}\left(D=D_{i}\right)=\frac{1}{N}$. When $\Delta Q_{D_{i}}^{G_{\theta}}$ is larger, that is, there is much room for $G_{\theta}$ to improve in the corresponding objective, AMPGAN will select $D_{i}$ with a larger probability.

Then the gradient of (8) can be computed as:

$$
\begin{aligned}
\nabla_{\theta} \mathcal{J}(\theta) & =\mathbb{E}_{D \sim \pi(Q)} \sum_{t=1}^{T} \mathbb{E}_{Y_{1: t-1} \sim G_{\theta}} \\
& {\left[\sum_{y \in Y} \nabla_{\theta} G_{\theta}\left(y_{t} \mid Y_{1: t-1}\right) Q_{D}^{G_{\theta}}\left(y_{t} \mid Y_{1: t-1}\right)\right] }
\end{aligned}
$$

We can use $\theta \leftarrow \theta+\alpha_{k} \nabla_{\theta} \mathcal{J}(\theta)$ to update the parameters $\theta$ of the generator, where $\alpha_{k}$ denotes the learning rate. And in practice, we estimate the expectation in the Eq. (10) by sampling discirminators according to $\pi(Q)$.



In summary, Algorithm 1 shows the details of AMPGAN. Before adversarial training, we first pretrain the generator $G_{\theta}$ using MLE on training set $\mathcal{S}$, and then use $G_{\theta}$ to generate negative examples. We sample positive examples from $\mathcal{S}$ and combine them with negative ones to pretrain parameterized discriminators $\left\{D_{i}\right\}_{i=1}^{N}$. During adversarial training, $G_{\theta}$ and each discriminator are trained alternately. At each training step, a discriminator $D_{i}$ will be selected from $\left\{D_{i}\right\}_{i=1}^{N}$ to participate in adversarial training according to distribution $\pi(Q)$. We sample positive examples from $\mathcal{S}$ and use $G_{\theta}$ to synthesize corresponding negative ones and use both to train $D_{i}$. $G_{\theta}$ is updated by using the policy gradient and MC search based on the expected final value function received from $D_{i}$. Finally, we adaptively adjust the sampling distribution $\pi(Q)$ based on the increments of the generator's scores in various aspects. Following [Li et al., 2017], we employ the Teacher Forcing strategy to tell $G_{\theta}$ what sequences are good and guide $G_{\theta}$ push itself to produce these good sequences by exposing the good target sentence to it directly.

\subsection{AMPGAN for Dialogue Generation}

We train the dialogue generator $G_{\theta}$ against 3 discriminators.

\section{Generator}

We use a standard SEQ2SEQ [Sutskever et al., 2014] model implemented by two-layer LSTMs with attention mechanism [Luong et al., 2015] to implement the generator's policy $G_{\theta}$ [Li et al., 2017]. $G_{\theta}$ encodes the input queries into hidden states, and then decodes them into output responses.

\section{Real-Fake Discriminator}

We continue the previous works [Li et al., 2017] and use the generic Real-Fake discriminator $D_{r f}$ to judge whether an input dialogue utterances $\{x, y\}$ are generated by humans (denoted as a real label) or machines (fake). $D_{r f}$ is essentially a two-classifier, and uses a hierarchical encoder [Serban et al., 
2016] with a two-class softmax layer to report the probability $Q_{+}$that $\{x, y\}$ are real and $Q_{-}$that $\{x, y\}$ are fake. The purpose of using $D_{r f}$ is to encourage $G_{\theta}$ to generate dialogue utterances that are difficult to distinguish between real or fake by taking $Q_{+}$as the reward.

\section{Syntactic Discriminator}

We notice that the generated sequences are often confusing because the arrangement of words is not in line with human syntax norms. We try to improve the syntax score of the generated sequences by feeding $G_{\theta}$ the syntactic reward reported by the proposed syntactic discriminator $D_{\text {syn }}$. Following [Vashishth et al., 2019], We use graph convolutional networks (GCN) to extract syntactic features embedding $e_{\text {syn }}$ of a sequence. We use the Stanford CoreNLP parser [Manning et $a l ., 2014]$ to parse each word sequence $s=\left\{x_{1}, x_{2}, \ldots, x_{|s|}\right\}$ into a dependency parse graph $\mathcal{G}=\left(\mathcal{V}_{s}, \mathcal{E}_{s}\right)$, whereas $\mathcal{V}_{s}=$ $\left\{x_{1}, x_{2}, \ldots, x_{|s|}\right\}$ and $\mathcal{E}_{s}$ stands for the labeled directed dependency edges $\left(x_{i}, x_{j}, l_{i j}\right)$, where $l_{i j}$ is the dependency relaiton label of $x_{i}$ to $x_{j}$. We use the corresponding word embedding $\boldsymbol{v}_{i} \in \mathbb{R}^{d}$ to initialize each node embedding $\boldsymbol{h}_{i}^{0} \in \mathbb{R}^{d}$ in $\mathcal{V}_{s}$.

In graph convolutional layers of $D_{\text {syn }}$, we employ the SynGCN [Vashishth et al., 2019] model with Edge Label Gating Mechanism to aggregate each node. Then embedding $\boldsymbol{h}_{i}^{k} \in \mathbb{R}^{d}$ of node $i$ after $k$ GCN layers is computed as $\boldsymbol{h}_{i}^{k}=\operatorname{Syn} G C N\left(\boldsymbol{h}_{i}^{0}\right)$. We use max-pooling operation to obtain syntactic embedding $\boldsymbol{e}_{s y n}=\left\{e_{m}\right\}_{m=1}^{d}$, where $e_{m}=\max \left\{\boldsymbol{h}_{i m}^{k}\right\}_{i=1}^{|s|}$. Finally, syntactic score $s_{s y n}$ is computed as: $s_{\text {syn }}=\sigma\left(B N\left(\boldsymbol{W}_{s} \boldsymbol{e}_{\text {syn }}+b_{s}\right)\right)$, where $\boldsymbol{W}_{s} \in \mathbb{R}^{1 \times d}$ is a parameter vector, $b_{s}$ is a bias, $B N$ is a batch normalization layer [Ioffe and Szegedy, 2015], and $\sigma(\cdot)$ is the sigmod function. We use $s_{s y n}$ as the reward to guide $G_{\theta}$ to produce utterances that conform to human expressions.

\section{Information Discriminator}

The generator tends to produce high frequency words. We design the information discirminator $D_{\text {info }}$ to directly compute the normalized information amount $s_{\text {info }}$ of the generated utterance with $n$ tokens. Formally, $s_{\text {info }}$ is computed as $s_{\text {info }}=\frac{1}{n} \sum_{i=1}^{n} \frac{I\left(w_{i}\right)-I\left(w_{\min }\right)}{I\left(w_{\max }\right)-I\left(w_{\min }\right)}$, whereas $I\left(w_{i}\right)=$ $-\log p\left(w_{i}\right), p\left(w_{i}\right)$ is the frequency of word $w_{i}, w_{\max }$ and $w_{\min }$ are the two words with the largest and smallest word frequencies in the corpus, respectively. We use $s_{\text {info }}$ as the reward to push $G_{\theta}$ to explore more novel words.

\section{Experiments}

\subsection{Datasets}

Cornell Movie Dataset* (denoted as $\mathcal{S}_{1}$ ) contains a large metadata-rich collection of fictional conversations extracted from raw movie scripts. It consists of 220579 exchanges between 10292 pairs of characters in the movie, involving 9035 characters in 617 movies, a total of 304713 utterances. OpenSubtitles Dataset $\left(\mathcal{S}_{2}\right)$ is a well-known human-human scripted dialogue dataset. It is extracted from movie subtitles which are not speaker-aligned [Tiedemann, 2009]. We use

\footnotetext{
${ }^{*}$ Cornell Movie-Dialogs Corpus
}

the English short message data, which contains about 140M utterances in total, covers $106 \mathrm{~K}$ movie works.

\subsection{Training Details}

We sort the words in each corpus by word frequency and use the first $35 \mathrm{~K}$ words with high word frequency as the vocabulary. We filter out dialogue utterances with less than 5 or more than 40 tokens. We set the training batch size to 128 , the size of word embeddings and the graph node embeddings to 512, the hidden size of hidden layers of all encoders in all models to 256, and the number of all LSTM layers to 2, and GCN layer number of $D_{s y n}$ to 1 . We train all models using Adam optimizer [Kingma and Ba, 2015], and all the dropout rates to 0.7 . For the generator $G_{\theta}$, we use the learning rate decay strategy and set the initial learning rate to 0.1 and the decay factor to 0.99 . We set the learning rate to 0.001 fixedly for $D_{r f}$ and $D_{s y n}$. In pre-training, we first pre-train $G_{\theta} 5000$ iterations, then use $G_{\theta}$ to produce $2500 \times 128$ negative examples and sample the same amount of positive ones from the dataset correspondingly. We combine both to pre-train $D_{r f}$ and $D_{\text {syn }}$. In MC search, we employ the experience [Li et $a l ., 2017]$ that given a partially decoded $s_{P}, G_{\theta}$ will keep sampling tokens in word distribution until decoding is complete. Repeat this process $k$ times ( $k$ is set to 7 ) and obtain $k$ sequences sharing a common prefix $s_{P}$. The average of the corresponding $k$ scores given by the discriminator is used as the reward. During training of each model, if the performance on the validation set has not improved for a long time, stop training and choose the checkpoint with the best performance.

\subsection{Experiments Results}

We use the following models as the baselines. (1) MLE trains a standard SEQ2SEQ generator $G_{\theta}$ using the traditional MLE; (2) PG-BLEU [Yu et al., 2017] trains $G_{\theta}$ by policy gradient using the BLEU score of generated sequences as the reward directly, thereby obtaining a higher BLEU score; (3) AdverREGS [Li et al., 2017] is a standard adversarial model that $G_{\theta}$ is trained against a real-fake discirminator alone, and can be viewed as an extension of SeqGAN on the SEQ2SEQ framework; (4) DP-GAN [Xu et al., 2018] designs a finegrained diversity discriminator to guide $G_{\theta}$ to produce sequences with high diversity. (5) DAL [Cui et al., 2019] tries to increase the response diversity by utilizing the duality between query and response generation.

We regularly combine different discriminators to guide the generator: (1) $D_{1}$ : only use single $D_{r f}$, noted as AdverREGS; (2) $D_{2}$ : only use single $D_{s y n}$; (3) $D_{3}$ : only use single $D_{\text {Info }}$; (4) $D_{1} \& D_{2}$ : combine $D_{r f}$ and $D_{\text {syn }}$; (5) $D_{1} \& D_{3}$ : combine $D_{r f}$ and $D_{I n f o}$; (6) $D_{2} \& D_{3}$ : combine $D_{s y n}$ and $D_{\text {Info }} ;$ (7) $D_{1} \& D_{2} \& D_{3}$ : combine $D_{r f}, D_{s y n}$ and $D_{\text {Info. }}$.

We use two categories of metrics to evaluate each model, namely Adversarial Evaluation [Li et al., 2017; Bowman et al., 2016] and widely used Common Evaluation metrics.

\section{Adversarial Evaluation}

Adversarial evaluation helps to analyze the impact of different discriminator combinations of AMPGAN. Following [Li et al., 2017], we train automated machine evaluators to distinguish machine-generated responses from human-generated 
responses, such as accurately distinguishing real or fake, giving high scores to real responses and low scores to the fake. We design three machine evaluators, namely RealE, SyntaxE and InfoE, corresponding to $D_{r f}, D_{s y n}$ and $D_{\text {info }}$, to report the ratio FoolRate of the cases that RealE are deceived, the syntax score SynScore, and the ratio InfoRate of the information amount of the generated responses to the ground truth, respectively. Note that each evaluator is in the same architecture as its corresponding discriminator, but trained separately using supervised methods and do not participate in adversarial training. The abilities of parameterized RealE and SyntaxE are 0.09 and 0.11, indicates how much we can trust the evaluation results given by the evaluators, and the smaller the value, the better. We also report the comprehensive score NormAve of SynScore, InfoRate and FoolRate as $\frac{1}{3} \sum_{i=1}^{3}\left(x_{j}^{i}-x_{\text {min }}^{i}\right) /\left(x_{\text {max }}^{i}-x_{\text {min }}^{i}\right)$, where $x_{j}^{i}, x_{\text {max }}^{i}$, and $x_{\text {min }}^{i}$ are the score of model $j$, maximum and minimum in the table on the $i$-th indicator respectively.

Table 1 shows the evaluation results. From Table 1 we can find out that AMPGAN can effectively improve the generator $G_{\theta}$. Specifically, it can be summarized as follows: (1) Using a single discriminator is risky. We can find each model can fool the real-fake evaluator RealE to a large extent (greater than $97 \%$ ) on $\mathcal{S}_{1}$, which shows that generators can easily perform well in this objective on $\mathcal{S}_{1}$, even without the guidance reward of $D_{r f}$. (2) The generators against a single discriminator can go deeper towards expected objectives. We can find the generator against single $D_{1}$ or $D_{2}$ or $D_{3}$ performs better in its corresponding objective than other single adversarial training models. (3) The generator trained against multiple discriminators can find a balance and achieve an overall improvement towards multiple objectives, although it may be weakened unilaterally. We can find the generator against $D_{1} \& D_{2} \& D_{3}$ achieves significantly higher overall performance, although unilateral indicator values decrease a bit. Empirically then, we recommend that designers should design a general objective with the corresponding discirminator to report the basic but universal reward and supplement it with other specific rewards according to actual needs, so that the model can perform well in general and also stand out in several areas.

\section{Common Evaluation}

The metrics of common evaluation are as follows:

(1) BLEU score [Papineni et al., 2002] is widely used in sequence generation tasks [Li et al., 2016], measuring the overlaping between the generated word sequences and the ground truth; (2) Distinctness metric proposed by [Li et al., 2016] measures the diversity of generated token sequences. (3) Human Evaluation (HM): We ask three human annotators with a linguistic background to report on the overall quality of the responses to 200 examples randomly sampled from the test set, which are generated by the full AMPGAN model and all baseline models. (4) Similarity\&Relevance: We also report the similarity between the ground truth responses and the generated, and the relevance between the queries and the generated responses at semantic level by calculating cosine similarity of the two token sequence embeddings computed by the pretrained BERT [Devlin et al., 2018] model.

Table 2 shows the evaluation results. From Table 2, we

\begin{tabular}{c|c|c|c|c|c}
\hline & Model & SynScore & InfoRate & FoolRate & NormAve \\
\hline \multirow{6}{*}{} & MLE & 0.624 & 0.817 & 0.984 & 0.367 \\
& PG-BLEU & 0.540 & 0.889 & 0.987 & 0.498 \\
& AdverREGS $\left(D_{1}\right)$ & 0.584 & 0.864 & 0.992 & 0.573 \\
& DP-GAN & 0.481 & 0.921 & 0.993 & 0.617 \\
$\mathcal{S}_{1}$ & DAL & 0.521 & 0.917 & 0.992 & 0.630 \\
\cline { 2 - 6 } & SynGCN $\left(D_{2}\right)$ & $\mathbf{0 . 7 1 5}$ & 0.832 & 0.979 & 0.403 \\
& InfoAmt $\left(D_{3}\right)$ & 0.377 & $\mathbf{0 . 9 5 0}$ & 0.987 & 0.474 \\
& $D_{1} \& D_{2}$ & 0.672 & 0.801 & $\mathbf{0 . 9 9 8}$ & 0.624 \\
& $D_{1} \& D_{3}$ & 0.541 & 0.896 & 0.993 & 0.620 \\
& $D_{2} \& D_{3}$ & 0.655 & 0.880 & 0.991 & 0.661 \\
& $D_{1} \& D_{2} \& D_{3}$ & 0.653 & 0.871 & 0.995 & $\mathbf{0 . 7 1 0}$ \\
\hline \multirow{6}{*}{$\mathcal{S}_{2}$} & MLE & 0.348 & 0.859 & 0.221 & 0.193 \\
& PG-BLEU & 0.356 & 0.875 & 0.270 & 0.250 \\
& AdverREGS $\left(D_{1}\right)$ & 0.392 & 0.861 & $\mathbf{0 . 6 5 5}$ & 0.600 \\
& DP-GAN & 0.428 & 0.932 & 0.351 & 0.536 \\
& DAL & 0.401 & 0.919 & 0.435 & 0.595 \\
\cline { 2 - 6 } & InfoACN $\left(D_{2}\right)$ & $\mathbf{0 . 5 0 0}$ & 0.832 & 0.360 & 0.466 \\
& $\left.D_{1} \& D_{2}\right)$ & 0.285 & $\mathbf{0 . 9 5 1}$ & 0.373 & 0.450 \\
& $D_{1} \& D_{3}$ & 0.495 & 0.822 & 0.529 & 0.562 \\
& $D_{2} \& D_{3}$ & 0.355 & 0.864 & 0.533 & 0.457 \\
& $D_{1} \& D_{2} \& D_{3}$ & 0.458 & 0.931 & 0.401 & 0.688 \\
& & 0.472 & 0.926 & 0.477 & $\mathbf{0 . 7 5 5}$ \\
\hline \multirow{6}{*}{} & & & &
\end{tabular}

Table 1: Results of Adversarial Evaluation on both datasets.

can find out that AMPGAN framework do help achieve better results on these common metrics. Specifically, the generator against three discriminators performs very well. It achieves the highest BLEU-1 score and Dist-1, Dist-2, and Dist-3 scores, as well as relatively higher BLEU-2 and Dist4 scores on both datasets, indicating that the generated token sequences have higher overlap with ground truth responses and higher diversity. It also achieve the highest ranking of Human Evaluation over baselines on both datasets. Moreover, the pairwise adversarial training generators also achieved higher word overlap and diversity than the corresponding single adversarial training ones separately.

Figure 2 shows the results of similarity\&relevance evaluation. We can clearly find the combination of $D_{1} \& D_{2} \& D_{3}$ makes the generated sentences obtain the highest similarity with ground truth responses and the highest relevance with queries on both datasets, indicating the token sequences generated by this generator are closest to the ground truth at the semantic level. Moreover, we can also find out the trend that the generator against multiple combined discriminators can achieve higher similarity and relevance than the one against each of these discriminators alone.

\section{Effectiveness of Adaptive Sampling Distribution}

We also investigate the adjustment of the discriminator sample distribution during training, as shown in Figure 3, and conduct a comparative experiment between using adaptive distribution and fixed uniform distribution, as shown in Table 3. From Figure 3, we can find out that different datasets have different characteristics, and AMPGAN can capture these characteristics by adaptively adjusting the sampling distribution of all objectives. And during training, improvement degrees of $G_{\theta}$ towards different objectives are constantly changing, so it is very useful to dynamically adjust the distribution. In addition, from Table 3, we can find that compared with using a fixed uniform distribution, using the adaptive adjustment distribution helps AMPGAN achieve higher scores of adversarial evaluation and diversity on two datasets, which also shows the effectiveness of the adaptive distribution. Tak- 


\begin{tabular}{|c|c|c|c|c|c|c|c|c|c|c|c|c|c|c|}
\hline \multirow{2}{*}{ Model } & \multicolumn{7}{|c|}{ Cornell } & \multicolumn{7}{|c|}{ Opensubtitles } \\
\hline & BLEU-1 & BLEU-2 & Dist-1 & Dist-2 & Dist-3 & Dist-4 & $\mathrm{HM}$ & BLEU-1 & BLEU-2 & Dist-1 & Dist-2 & Dist-3 & Dist-4 & HM \\
\hline MLE & 12.8 & 1.54 & 34.8 & 62.4 & 78.6 & 92.4 & 5.33 & 14.4 & 2.42 & 28.4 & 53.1 & 73.8 & 91.0 & 5.67 \\
\hline PG-BLEU & 13.2 & 1.79 & 37.0 & 65.2 & 83.2 & 97.5 & 5.00 & 15.2 & 1.97 & 30.3 & 56.7 & 78.4 & 95.8 & 4.33 \\
\hline $\operatorname{AdverREGS}\left(D_{1}\right)$ & 12.1 & 1.91 & 38.3 & 65.0 & 82.8 & 95.6 & 4.33 & 14.5 & 2.30 & 30.3 & 56.5 & 78.1 & 95.7 & 4.00 \\
\hline DP-GAN & 12.0 & 1.60 & 37.8 & 66.9 & 84.2 & 98.3 & 2.00 & 14.7 & 1.92 & 30.5 & 56.9 & 79.1 & 97.6 & 2.33 \\
\hline DAL & 12.9 & 1.95 & 38.7 & 66.2 & 83.5 & 97.9 & 3.00 & 14.4 & 2.35 & 31.1 & 57.1 & 79.5 & 97.3 & 2.67 \\
\hline $\operatorname{SynGCN}\left(D_{2}\right)$ & 12.3 & 2.17 & 32.9 & 65.6 & 83.2 & 95.5 & - & 13.8 & 2.36 & 30.1 & 56.6 & 79.0 & 95.2 & - \\
\hline $\operatorname{InfoAmt}\left(D_{3}\right)$ & 11.8 & 2.02 & 36.9 & 64.7 & 83.1 & 95.5 & - & 14.3 & 2.42 & 30.8 & 57.3 & 79.4 & 96.4 & - \\
\hline$D_{1} \& D_{2}$ & 12.6 & 2.36 & 35.8 & 66.5 & 83.5 & 97.8 & - & 14.7 & 2.40 & 31.7 & 58.7 & 80.3 & 97.2 & - \\
\hline$D_{1} \& D_{3}$ & 12.9 & 2.14 & 38.0 & 66.1 & 83.7 & 97.5 & - & 14.9 & 2.45 & 32.4 & 58.2 & 80.0 & 97.4 & - \\
\hline$D_{2} \& D_{3}$ & 13.5 & 2.22 & 38.8 & 67.1 & 84.7 & 98.5 & - & 14.8 & 2.48 & 32.7 & 59.2 & 80.8 & 97.8 & - \\
\hline$D_{1} \& D_{2} \& D_{3}$ & 13.9 & 2.21 & 42.9 & 70.4 & 87.9 & 98.4 & 1.33 & 15.2 & 2.43 & 33.2 & 60.3 & 81.1 & 97.6 & 2.00 \\
\hline
\end{tabular}

Table 2: Results of common metrics evaluation on Cornell dataset and Opensubtitles dataset.


Figure 2: Results of similarity (sim) and relevance (rel) evaluation on the generated sentences by the generators against different discriminator combinations.

\begin{tabular}{c|c|c|c|c|c|c}
\hline \multirow{2}{*}{ Sample } & \multicolumn{3}{|c|}{ Cornell } & \multicolumn{3}{c}{ Opensubtitles } \\
\cline { 2 - 7 } & NormAve & BAve & DAve & NormAve & BAve & DAve \\
\hline Uniform & 0.679 & 7.89 & 73.8 & 0.698 & $\mathbf{8 . 8 4}$ & 67.5 \\
Adaptive & $\mathbf{0 . 7 1 0}$ & $\mathbf{8 . 0 6}$ & $\mathbf{7 4 . 9}$ & $\mathbf{0 . 7 5 5}$ & 8.82 & $\mathbf{6 8 . 1}$ \\
\hline
\end{tabular}

Table 3: Comparison of two sampling distributions. BAve is the average score of BLEU-1 and BLEU-2, and DAve is the average score of Dist-1, Dist-2, Dist-3 and Dist-4.

ing the OpenSubtitles curve as an example, when $G_{\theta}$ has been promoted sufficiently towards one objective and its improvement room becomes relatively small, then AMPGAN will reduce its probability and focus on other objectives, so as to achieve the purpose of balancing all objectives and improving overall performance. And because $D_{\text {info }}$ is absolutely accurate and can basically not be deceived by $G_{\theta}, D_{\text {info }}$ can always provide reward to guide $G_{\theta}$ to improve, which is reflected in its high probabilities. However, the $D_{r f}$ has the risk of saturation [Xu et al., 2018]. From Cornell curve, we can find that there is no improvement room towards the objective of $D_{r f}$ soon, indicating that $G_{\theta}$ can quickly fool $D_{r f}$.

\section{Related Works}

SeqGAN [Yu et al., 2017] successfully employs the promising GAN framework into the discrete sequence generation task by utilizing policy gradient and MC search. AdverREGS [Li et al., 2017] employs adversarial training to guide the generator to produce dialogue utterances that difficult for evaluators to distinguish between human-generated and machinegenerated. Many models focus on increasing the diversity of the generated responses: DP-GAN [Xu et al., 2018] designs a fine-grained discriminator which has a better diversity evaluation method; The Adversarial Information Maximiza-
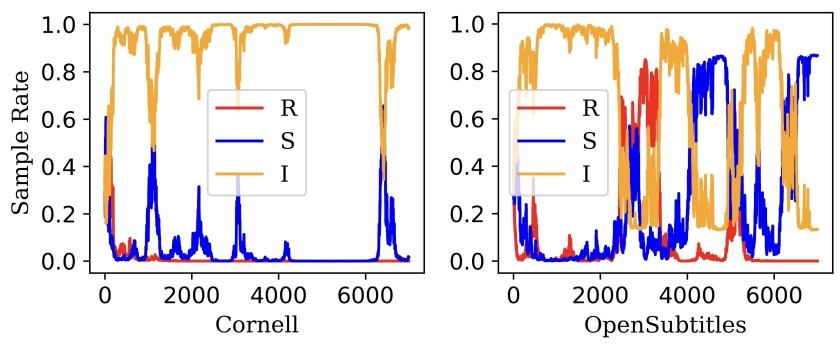

Figure 3: Adaptive adjustment of sampling distribution of RealFake(R), SynGCN(S) and InfoAmt(I) as the training progresses.

tion (AIM) [Zhang et al., 2018] optimizes a variational lower bound on pairwise mutual information between query and response; And DAL [Cui et al., 2019] utilizes the duality between query generation and response generation.

Recent literatures have demonstrated promising results in image generation tasks for extending the basic GAN framework using multiple discriminators to train against the generator. D2GAN [Nguyen et al., 2017] combines KL and inverse $\mathrm{KL}$ divergence into a unified objective function and uses the complementary statistical characteristics of these divergences to effectively disperse the estimated density to capture multiple modes. The Generative Multi-Adversarial Nets (GMAN) [Durugkar et al., 2017] trains the generator against a softmax weighted arithmetic average of $K$ different discriminators in the same model architecture. HVM [Albuquerque et $a l ., 2019]$ proposes hypervolume maximization to efficiently optimize a weighted loss from all discriminators for the case of large neural networks.

\section{Conclusions}

In this paper, we propose the adaptively multi-objective adversarial dialogue generation framework using policy gradient, called AMPGAN, to push the generator to improve itself towards multiple objectives of linguistics by feeding these reward signals from the discriminators corresponding to these objectives. Comparative experimental results of different combination models and baseline models show that AMPGAN can significantly improve overall performance with only minor sacrifices in each objective.

\section{Acknowledgments}

This work was supported by the National Science Foundation of China (NSFC No. 61876196). 


\section{References}

[Albuquerque et al., 2019] Isabela Albuquerque, João Monteiro, Thang Doan, Breandan Considine, Tiago H. Falk, and Ioannis Mitliagkas. Multi-objective training of generative adversarial networks with multiple discriminators. In ICML, 2019.

[Bowman et al., 2016] Samuel R. Bowman, Luke Vilnis, Oriol Vinyals, Andrew Dai, Rafal Jozefowicz, and Samy Bengio. Generating sentences from a continuous space. In SIGNLL, 2016.

[Cui et al., 2019] Shaobo Cui, Rongzhong Lian, Di Jiang, Yuanfeng Song, Siqi Bao, and Yong Jiang. DAL: Dual adversarial learning for dialogue generation. In $N A A C L$ Workshop on NeuralGen, 2019.

[Deb, 2001] Kalyanmoy Deb. Multi-objective optimization using evolutionary algorithms, volume 16 . John Wiley \& Sons, 2001.

[Devlin et al., 2018] Jacob Devlin, Ming-Wei Chang, Kenton Lee, and Kristina Toutanova. Bert: Pre-training of deep bidirectional transformers for language understanding. arXiv preprint arXiv:1810.04805, 2018.

[Durugkar et al., 2017] Ishan P. Durugkar, Ian Gemp, and Sridhar Mahadevan. Generative multi-adversarial networks. In ICLR, 2017.

[Goodfellow et al., 2014] Ian Goodfellow, Jean PougetAbadie, Mehdi Mirza, Bing Xu, David Warde-Farley, Sherjil Ozair, Aaron Courville, and Yoshua Bengio. Generative adversarial nets. In NIPS, 2014.

[Ioffe and Szegedy, 2015] Sergey Ioffe and Christian Szegedy. Batch normalization: Accelerating deep network training by reducing internal covariate shift. In ICML, 2015.

[Kingma and Ba, 2015] Diederik P. Kingma and Jimmy Ba. Adam: A method for stochastic optimization. In ICLR, 2015.

[Li et al., 2016] Jiwei Li, Michel Galley, Chris Brockett, and Jianfeng Gao. A diversity-promoting objective function for neural conversation models. In NAACL, 2016.

[Li et al., 2017] Jiwei Li, Will Monroe, Tianlin Shi, Sébastien Jean, Alan Ritter, and Dan Jurafsky. Adversarial learning for neural dialogue generation. In EMNLP, 2017.

[Luan et al., 2016] Yi Luan, Yangfeng Ji, and Mari Ostendorf. Lstm based conversation models. arXiv preprint arXiv:1603.09457, 2016.

[Luong et al., 2015] Thang Luong, Hieu Pham, and Christopher D Manning. Effective approaches to attention-based neural machine translation. In EMNLP, 2015.

[Manning et al., 2014] Christopher Manning, Mihai Surdeanu, John Bauer, Jenny Finkel, Steven Bethard, and David McClosky. The Stanford CoreNLP natural language processing toolkit. In ACL: System Demonstrations, 2014.

[Neyshabur et al., 2017] Behnam Neyshabur, Srinadh Bhojanapalli, and Ayan Chakrabarti. Stabilizing gan training with multiple random projections. arXiv preprint arXiv:1705.07831, 2017.
[Nguyen et al., 2017] Tu Nguyen, Trung Le, Hung Vu, and Dinh Phung. Dual discriminator generative adversarial nets. In NIPS, 2017.

[Papineni et al., 2002] Kishore Papineni, Salim Roukos, Todd Ward, and Wei-Jing Zhu. Bleu: a method for automatic evaluation of machine translation. In ACL, 2002.

[Serban et al., 2016] Iulian V Serban, Alessandro Sordoni, Yoshua Bengio, Aaron Courville, and Joelle Pineau. Building end-to-end dialogue systems using generative hierarchical neural network models. In $A A A I, 2016$.

[Somesh Das Gupta, 2000] Jun Shao. Somesh Das Gupta. Mathematical statistics. Springer, 2000.

[Sutskever et al., 2014] I Sutskever, O Vinyals, and QV Le. Sequence to sequence learning with neural networks. NIPS, 2014.

[Sutton et al., 2000] Richard S Sutton, David A McAllester, Satinder P Singh, and Yishay Mansour. Policy gradient methods for reinforcement learning with function approximation. In NIPS, 2000.

[Tao et al., 2018] Chongyang Tao, Shen Gao, Mingyue Shang, Wei Wu, Dongyan Zhao, and Rui Yan. Get the point of my utterance! learning towards effective responses with multi-head attention mechanism. In IJCAI, 2018.

[Tiedemann, 2009] Jörg Tiedemann. News from opus-a collection of multilingual parallel corpora with tools and interfaces. 2009.

[Vashishth et al., 2019] Shikhar Vashishth, Manik Bhandari, Prateek Yadav, Piyush Rai, Chiranjib Bhattacharyya, and Partha Talukdar. Incorporating syntactic and semantic information in word embeddings using graph convolutional networks. In $A C L, 2019$.

[Xu et al., 2018] Jingjing Xu, Xuancheng Ren, Junyang Lin, and $\mathrm{Xu}$ Sun. Diversity-promoting GAN: A cross-entropy based generative adversarial network for diversified text generation. In EMNLP, 2018.

[Yu et al., 2017] Lantao Yu, Weinan Zhang, Jun Wang, and Yong Yu. Seqgan: Sequence generative adversarial nets with policy gradient. In $A A A I, 2017$.

[Zhang et al., 2018] Yizhe Zhang, Michel Galley, Jianfeng Gao, Zhe Gan, Xiujun Li, Chris Brockett, and Bill Dolan. Generating informative and diverse conversational responses via adversarial information maximization. In NeurIPS, 2018. 